\title{
REVIEW OF ADVANCES IN COBB ANGLE CALCULATION AND IMAGE-BASED MODELLING TECHNIQUES FOR SPINAL DEFORMITIES
}

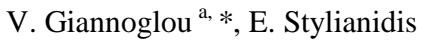 \\ ${ }^{\text {a }}$ School of Urban-Regional Planning and Development Engineering, Aristotle University of Thessaloniki, GR 54124 - \\ vgiannog@gmail.com \\ ${ }^{a}$ School of Urban-Regional Planning and Development Engineering, Aristotle University of Thessaloniki, GR 54124 - sstyl@auth.gr
}

Commission V, WG V/5

KEY WORDS: Scoliosis, Spine, Cobb angle, X-rays, 3D model, Image based techniques.

\begin{abstract}
:
Scoliosis is a 3D deformity of the human spinal column that is caused from the bending of the latter, causing pain, aesthetic and respiratory problems. This internal deformation is reflected in the outer shape of the human back. The golden standard for diagnosis and monitoring of scoliosis is the Cobb angle, which refers to the internal curvature of the trunk. This work is the first part of a postdoctoral research, presenting the most important researches that have been done in the field of scoliosis, concerning its digital visualisation, in order to provide a more precise and robust identification and monitoring of scoliosis. The research is divided in four fields, namely, the X-ray processing, the automatic Cobb angle(s) calculation, the 3D modelling of the spine that provides a more accurate representation of the trunk and the reduction of X-ray radiation exposure throughout the monitoring of scoliosis. Despite the fact that many researchers have been working on the field for the last decade at least, there is no reliable and universal tool to automatically calculate the Cobb angle(s) and successfully perform proper 3D modelling of the spinal column that would assist a more accurate detection and monitoring of scoliosis.
\end{abstract}

\section{INTRODUCTION}

Scoliosis is defined as the greater than 10 degrees curvature of the spine, as measured on an X-ray. Anything less is considered to be normal variation. The most common type of scoliosis, idiopathic scoliosis, has no specific identifiable cause so far. Around $2 \%$ of the people are diagnosed with scoliosis worldwide, whereas if a family member is diagnosed with scoliosis, other members have percentage as high as $20 \%$ to develop scoliosis (Department of Orthopaedic Surgery, http://orthosurg.ucsf.edu/patient-

care/divisions/spine/conditions/deformity/scoliosis/), which comprises strong evidence that it is inherited. Scoliosis is found at any age, but it is most common in children over 10 years old. Scoliosis can be treated and healed: treatment aims at improving the health and the aesthetics of the patients and it is of paramount importance for the health and self-esteem of juveniles.

If the doctor suspects a scoliosis case, after measuring the trunk asymmetry with the scoliometer, an X-ray of the back will be taken, from which the doctor measures the Cobb angle, which is the golden standard to quantify and grade scoliosis. Being manually executed, Cobb angle calculation entails subjectivity, errors and it is time consuming.

Considering the severity of scoliosis, authors gathered the most important researches in various fields of the subject, pointing out their strengths and their weaknesses, so that other researchers, as well as the authors themselves, can have a solid ground from where to begin their works.
The most reliable means of identifying a scoliotic patient is the $\mathrm{X}$-ray, therefore the initial field of the review is the $\mathrm{X}$-ray processing. With the development of image processing procedures, X-ray images have also been affected, exhibiting some very appealing methodologies to cope with, as it will be explained in more detail later.

Another very crucial field in the advances of spinal deformities is the Cobb angle. Being the current golden standard, it is important to be accurately calculated and other researchers strove in finding computerised methodologies to do so as it will be presented in the main part of this work.

As the Cobb angle is a 2D measurement of a 3D deformity, it is not the best indicator of the severity manifested in external appearance. The $3 \mathrm{D}$ representation of the human spinal column that would successfully depict the real nature of scoliosis is a field that contains tons of work done. To this end, many researchers occupied themselves to develop methodologies for $3 \mathrm{D}$ representation of the spine.

While all the above mentioned efforts have distinctive characteristics that make them valuable to the scientific community of scoliosis, what is missing is a uniform platform that encompasses all the above features, providing an inclusive tool that is able to perform all the necessary actions (spine detection, Cobb angle calculation, 3D model of the spine) in order to provide maximum insight for the caretaker of the patient. Authors reckon that a platform like this is the next step based on all the advances that were reviewed in this work.

\footnotetext{
* Corresponding author
} 
Since this work is part of a post-doctoral study, the reviewed papers are oriented towards the research goals of the aforementioned study. It is well understood that are plenty more interesting sub-fields in scoliosis subject, but it would be impossible to present all the related studies in a limited space research paper.

The rest of the paper is organised as follows: Section 2 is the main part of the present work, including all the paper reviews that comprise the basis of knowledge for the future work. In particular, subsection 2.1 analyses X-ray processing techniques that constitute the first step for detecting spinal deformities in human back. Subsection 2.2 elaborates on the findings concerning Cobb angle detection in literature. Subsection 2.3 deals with the 3D reconstruction of the spinal column techniques and subsection 2.4 focuses on scoliosis assessment techniques that include less or no radiation at all. In section 3 there is a discussion on the findings presented in this paper and also refers to the potential work that could commence from the aforementioned findings.

\section{LITERATURE REVIEW}

\subsection{X-ray processing}

$\mathrm{X}$-ray processing has been an open issue for a long time and will continue to be a crucial part of spine deformation field, due to the continuously evolving image processing methods. In 2009, Benjelloun and Mahmoudi tried to identify the location and the orientation of the cervical vertebrae (Benjelloun \& Mahmoudi, 2009). They implemented an automatic corner points of interest detection method. Corners in digital images are the robust features that provide important information of objects, they give important clues for shape representation and analysis, while object information can be represented in terms of its corners. The aforementioned authors made use of "Harris" corner detector which is strongly invariant to rotation, scale, illumination variation, and image noise. The sequence of the procedure steps to complete the corner detection, are as follows: (a) image acquisition, (b) corner detection, (c) extracting of the corners belonging to vertebra left sides, (d) global estimation of the spine curvature, and (e) anterior face vertebra detection. Below is an example image (Figure 1) of this specific work.

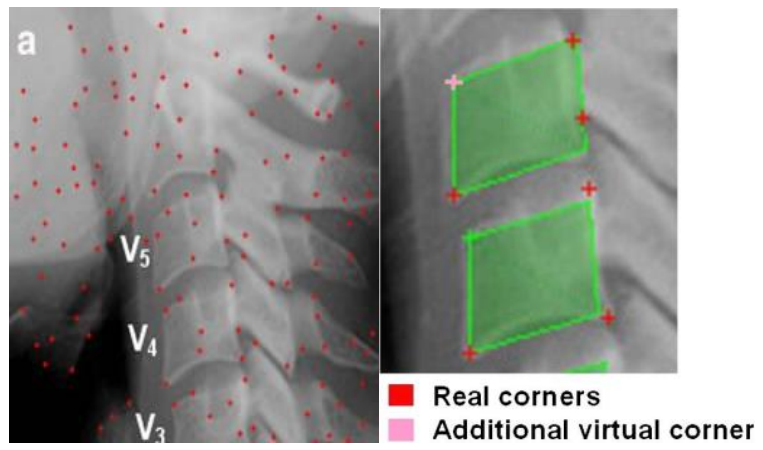

Figure 1. (a) Corner detection results using the Harris detector.

(b) Adding a virtual anterior face corner (if this one is not detected); (Source: Benjelloun \& Mahmoudi, 2009)

In another work (Duong et al., 2010), the authors attempted to automatically detect the spinal curve from conventional radiographs. Initially, the region of interest (ROI) was manually divided in three levels and an adapted filter was applied to denoise the image in each vertebral level. Texture descriptors were trained with Support Vector Machines (SVM) classifier, providing regions with spine-detected parts of the $\mathrm{X}$-ray. Finally, a spline curve is fitted through the centers of the predicted vertebral regions (Figure 2).

\subsection{Cobb angle calculation}

Being the golden standard and the most reliable and easy method -so far- to access the scoliosis severity, Cobb angle calculation is important to be able to be calculated in a fast and efficient way. To this end, quite a few researchers focused their scientific effort to develop automatic and computerised methods for the proper measurement of Cobb angle calculation by using different approaches.

Zhang et al. (Zhang et al., 2009) made a very interesting work, implementing a technique to automatically calculate the Cobb angle from a radiograph (X-ray) with the aid of the fuzzy Hough Transform (FHT).

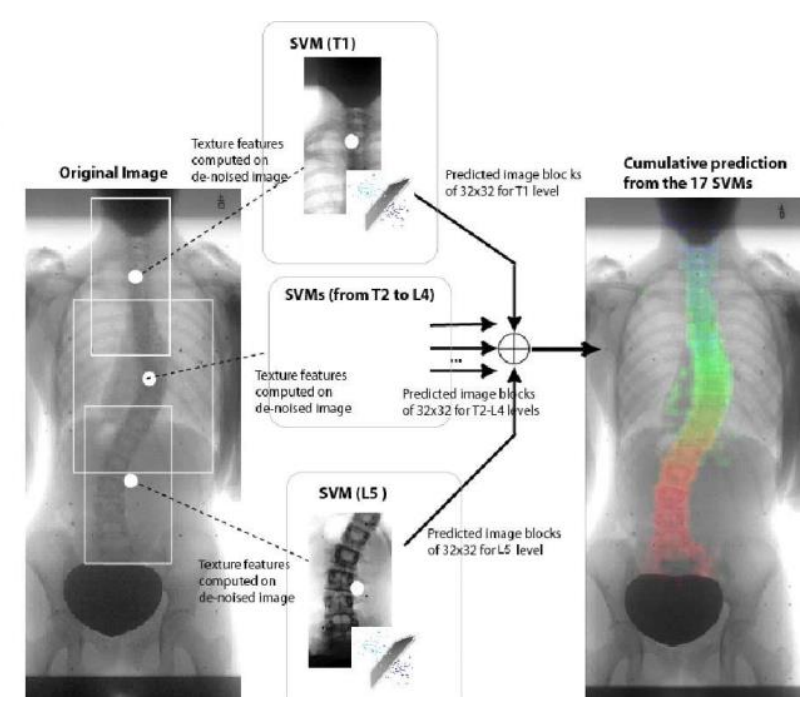

Figure 2. Block diagram of the segmentation algorithm. Each

ROI is divided into image blocks and texture features are computed from each image block; (Source: Duong et al., 2010)

More particularly, the authors firstly processed the X-ray to ameliorate the quality adjusting the brightness and contrast, and properly resizing the image. Afterwards the region of interest (ROI) was manually selected and the resized image was denoised by anisotropic diffusion. Canny operator was later used to detect the spinal edges and, finally, FHT with shape constraint was utilised to detect the direction of the vertebra. The whole procedure is depicted in (Figure 3). The proposed method was tested and compared with the manual measurement method to evaluate its performance. The manual method was measured by an orthopedic surgeon specialized in scoliosis. Two examiners were respectively asked to measure the Cobb angle on each radiograph by using the developed software twice. The overall performance of the automatic Cobb angle calculation method was quite satisfying, since the automatic measurement never declined more than $5^{\circ}$ from the manual one.

In 2010, Lawrence and Rinsky (Lawrence \& Rinsky, 2010) confirmed through their commentary two things. First that 
computerised measurement of the Cobb angle is growing fast and second that Cobb angle measurement for quantifying scoliosis has its deficiencies, being two-dimensional. The title they chose for their commentary says it all: Quantifying scoliosis: we are still not there.

Kundu et al. (Kundu et al., 2012), presented an interesting semiautomatic Cobb angle calculation methodology that begins by manually selecting the extreme vertebrae which tilt more towards the spine curve. Then authors implemented the Euclidean trimmed-mean filter for denoising the initial image followed by the suitable application of Otsu's method for automatic thresholding selection from the image, improving the performance of the later applied Canny edge detection technique (Canny, 1986). Finally, Hough transform is utilized for the vertebra slope detection and the measurement of the Cobb angle automatically takes place (Figure 4).

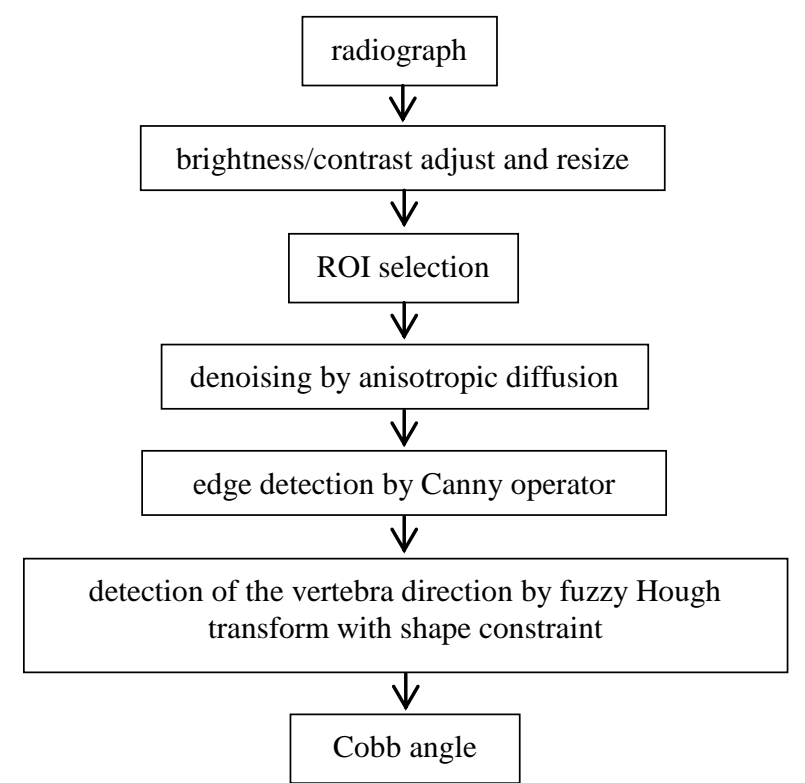

Figure 3. Automatic Cobb angle calculation procedure; Source: (Zhang et al., 2009)

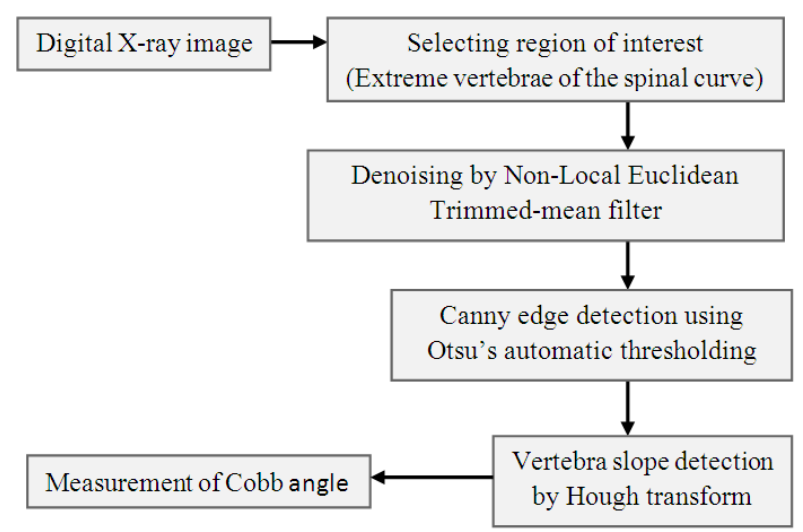

Figure 4. Flow diagram of Cobb angle measurement technique; (Source: Kundu et al., 2012)

In a very recent study, Sardjono et al. (Sardjono et al., 2013) used a modified charged particle model for determining the spinal curve on radiographical images. The Cobb angle was calculated out of three curve fitting methods implemented by the authors, namely, piecewise linear, splines and polynomials. Automatic calculations were then compared to manual ones performed by three specialists, and the result was very encouraging towards the automatic methods of piecewise linear and the polynomial techniques.

\subsection{D representation of the spine}

As stated before, Cobb angle is a measure of the 2D deviations of the spine, while scoliosis is a $3 \mathrm{D}$ deformity. Thus, researchers have turned their interest in finding means of measuring the severity of a falsely curved trunk that take into account the third dimension too. To this end, the first step is to find a way to transfer the 2D radiological images (AP and LAT $\mathrm{X}$-rays), that still comprise the basis of spine deformities, into the 3D space. Markelj et al. (Markelj, 2012), published a review paper for the $3 \mathrm{D} / 2 \mathrm{D}$ registration methods according to image modality, dimensionality and the nature of registration basis.

In the field of 3D representation of the spine, Delorme et al. (Delorme et al., 2003) applied high-resolution generic 3D information to the personalised $2 \mathrm{D}$ radiographs, creating a $3 \mathrm{D}$ model of spine and ribs (Figure 5).

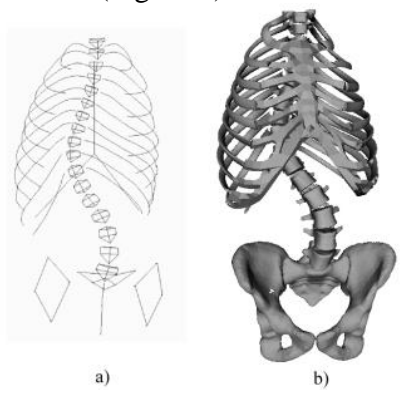

Figure 5. (a) 3D reconstructed geometry of the spine, the pelvis, and the rib cage. (b) High-resolution 3D model of a scoliotic subject's skeletal trunk; Source: (Delorme et al., 2003)

More particularly, the authors of the aforementioned work merged 2D radiological images from three positions (LAT, PA_0, PA_20) with CT-scan reconstructed anatomical primitives, which is high resolution generic 3D data (Figure 6). The developed technique involves application of known closerange photogrammetry techniques such as the Direct Linear Transformation (DLT) and 3D geometrical kriging to the problem of 3D Free Form Deformation (FFD) with unstructured control points. 


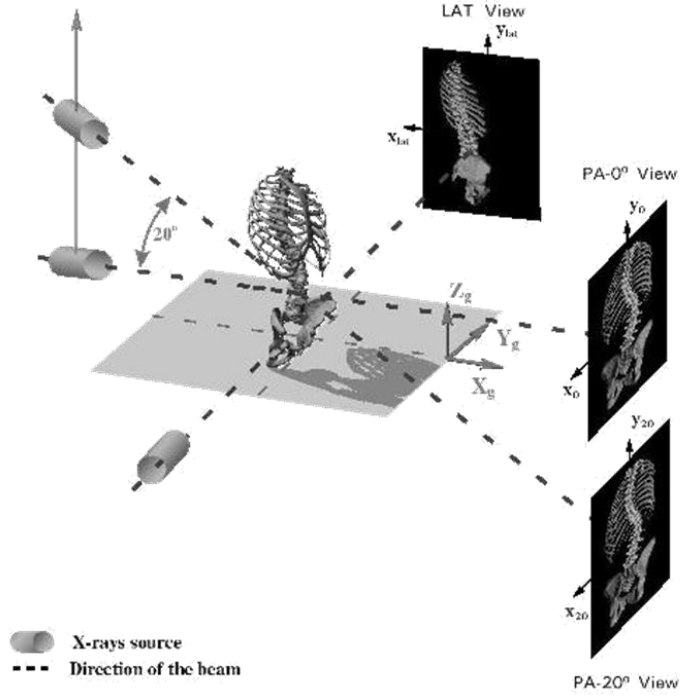

Figure 6. Three-dimensional radiographic images acquisition setup; Source: (Delorme et al., 2003)

In another work, Benameur et al. (Benameur et al., 2005) in their effort to develop a 3D reconstruction of the spine, made use of PA and LAT X-rays, as well as a priori hierarchical global knowledge on the geometric structure of the whole spine and the geometric structure of each vertebra (Figure 7).

In the rough geometric template for the crude spine registration, non-linear admissible global and local deformations are defined in order to create a 3D bi-planar reconstruction of the scoliotic Spine (Figure 8).

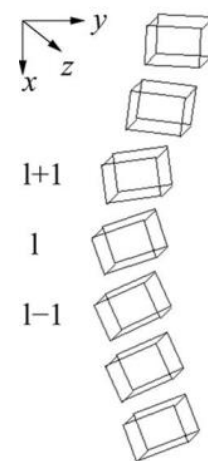

(a)
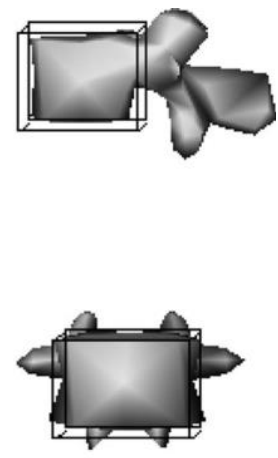

(b)

Figure 7. Crude prior model of the spine. (a) Deformable model of the whole spine and (b) cubic template representation associated with each vertebra; Source: (Benameur et al., 2005)

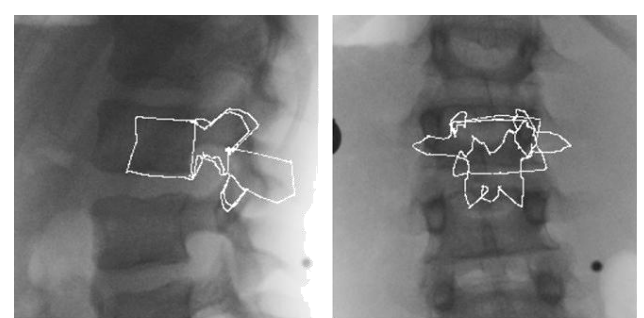

(a)

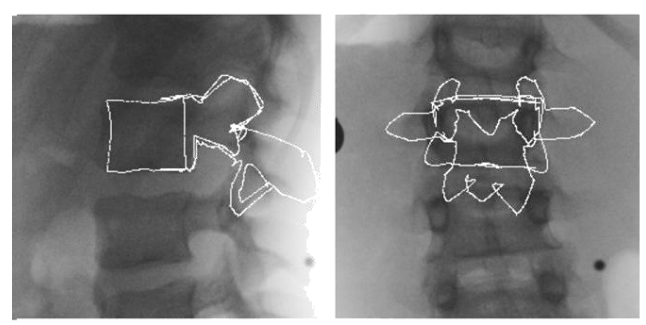

(b)

Figure 8. Projection of vertebra template on corresponding vertebra PA and LAT views (a) Initial (b) after deformations; Source: (Benameur et al., 2005)

In their work, Kadoury et al. (Kadoury et al., 2007) presented a very interesting work about $3 \mathrm{D}$ reconstruction of the spine and pelvis using a portable calibration object. More specifically, they made use of two radiographic images (PA, LAT) and a calibration object placed in the lumbar region during X-ray as a reference point (Figure 9).

Using the so-called weak-perspective algorithm, there was an initial approximation of the geometric parameters, while calibration information and six anatomical landmarks on each vertebra was identified on the radiograph and matched on both views (PA and LAT) to obtain the desired 3D coordinates using a stereo-triangulation algorithm. The latter is an algorithm to reconstruct a 3D model of the spine from the set of identified and matched anatomical landmarks on both X-rays. To do this, there is a calculation of the intersection point of the two X-rays from two homologous points, which are computed from a projection matrix and the $2 \mathrm{D}$ image anatomical landmark points. The intersection point between the two $\mathrm{x}$-rays corresponds to the $3 \mathrm{D}$ reconstruction of the two respective points.

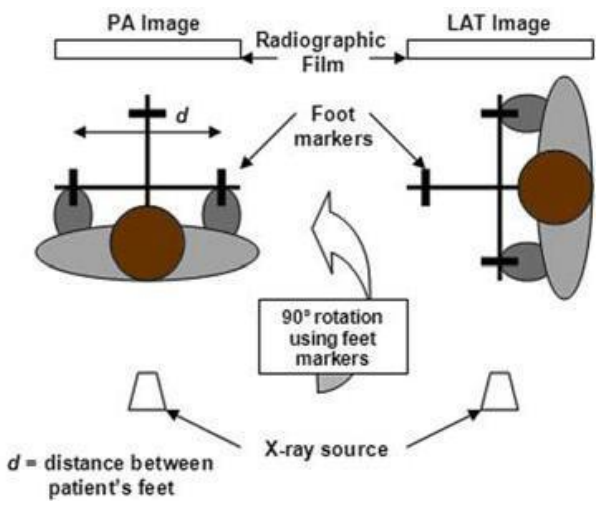

Figure 9. Positioning of the patient; Source: (Kadoury et al., 2007) 
Kadoury et al. later evolved their previous work (Kadoury et al., 2009), developing a personalised X-ray 3D reconstruction of the scoliotic spine from hybrid statistical and image-based models. Their research involved a 3D reconstruction method of the scoliotic spine using prior vertebra models and image-based information from bi-planar X-rays. Firstly, a global modelling approach was used by exploiting the 3D scoliotic curve reconstructed from a coronal and sagittal $\mathrm{x}$-ray image, in order to generate an approximate statistical model from a 3D database of 732 scoliotic spines. The $3 \mathrm{D}$ reconstruction of the spine is then achieved with a segmentation method in order to segment and isolate individual vertebrae on the radiographic planes, using 3D Fourier descriptors.

One year later, the same authors (Kadoury et al., 2010) applied self-calibration of bi-planar radiographic images through geometric spine shape descriptors. So far, calibration methods comprised either some cumbersome apparatus or manually identified landmarks that entailed errors. In this work, after a first enhancement of the X-ray image with filters, the spinal shape silhouettes and centre-line of the spine were extracted and the correspondence of $2 \mathrm{D}$ radiographs and $3 \mathrm{D}$ spine shape was calculated through the visual Hull reconstruction technique (Figure 10).

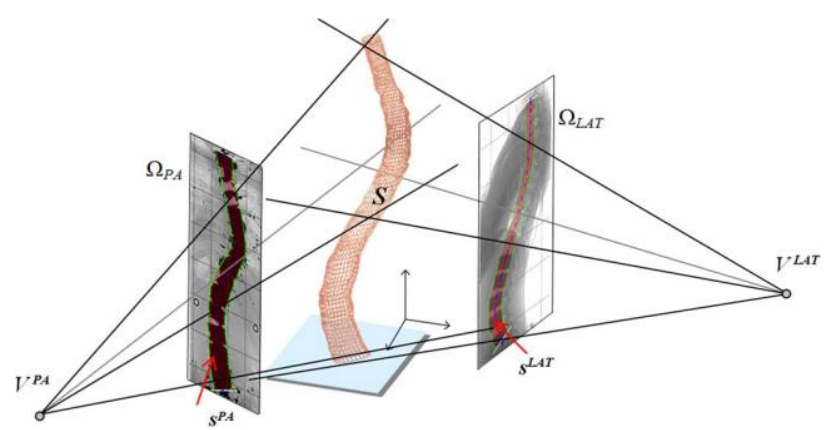

Figure 10. The shape of the object "S" is estimated by the intersection of both visual cones issued from viewpoints $\mathrm{V}$, offering an approximate representation of the global spine shape; Source: (Kadoury et al., 2010)

\subsection{Reducing X-ray exposure for scoliosis assessment}

Knott et al. (Knott et al., 2012), published a consensus paper in which authors analyse the side effects of $\mathrm{x}$-ray exposure in the paediatric population as it relates to scoliosis evaluation and treatment. Apart from the $5^{\circ}$ intra-observer and the $6.5^{\circ}$ interobserver variability, radiation exposure is found to significantly increase the breast cancer percentage four to ten times in females with scoliosis. As such, the aforementioned paper highlights the importance of developing alternative, radiationfree methods for assessing the severity of scoliosis and ended up to ten consensus statements that were presented and then voted. One statement among them sets the some limits in X-ray exposure depending on the scoliotic patient's age:

- For patients 0-5 years of age with early onset scoliosis: every 6 months

- For patients 6-12 years of age with juvenile scoliosis: every 6 months

- For patients 13-18 years of age with AIS, Risser Stage 0-1: every 12 months
- For patients 13-18 years of age with AIS, Risser Stage 2-3: every 12 months

- For patients 13-18 years of age with AIS, Risser Stage 4-5: every 18 months

- For patients 19-30 years of age with AIS, Post-growth surveillance: every 24 months

Taking into account the importance of developing alternative radiation-free methods for assessing the spine curvature, authors reviewed some works that focus not on the X-ray radiographs, but on indices on the surface back. More particularly, in 2006 (Patias et al., 2006), authors, taking advantage of a multicamera system setup that depicts the trunk from different views (Figure 11) and using an automatic image matching, they computed the 3D coordinates of a vast amount of surface points (Figure 12). In order for the photogrammteric 3D data of the back surface to have clinical relevance and to be practically useful to medical society, a number of indices were proposed that are linked to the spine shape. Therefore, the aforementioned methodology comprises a radiation-free approach to the assessment of scoliosis.

In another radiation-free work concerning scoliosis, Frerich et al. (Frerich et al, 2012) tested the Formetric 4D (http://www.diers.de/ProductPage.aspx?p=2) surface topography system that is widely used the last years in the scoliosis field. That system projects stripes of white light (raster lines) on the back of a standing patient and captures a digital photo of the image to assess pinpoint surface asymmetry and identify bony landmarks. The Formetric 4D was found comparable to radiography. Despite the fact that this device does not exactly predict curve magnitude, the predictions correlate strongly with the Cobb angles determined from radiographs.
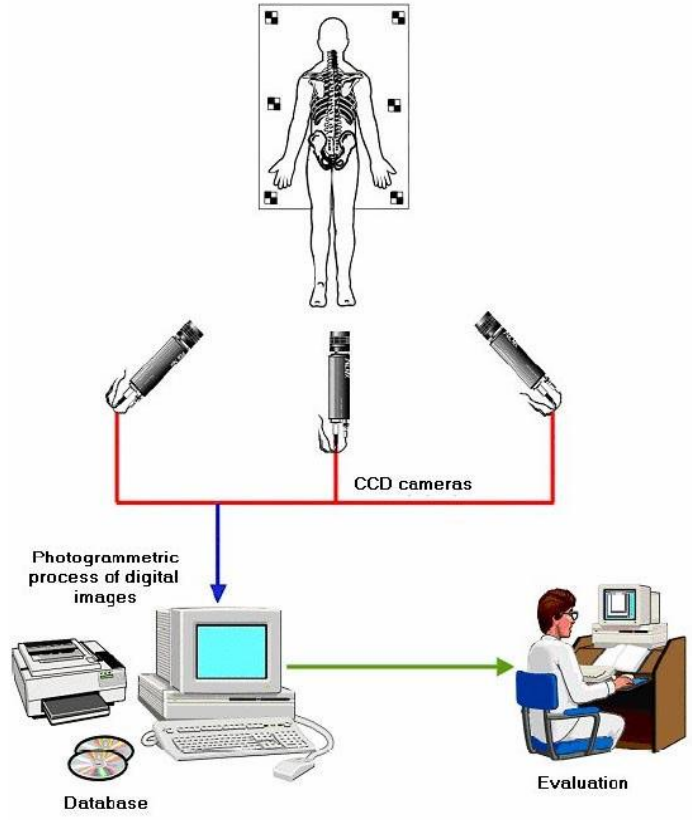

Figure 11. The proposed three-camera system followed by software analysis; Source: (Patias et al., 2006) 


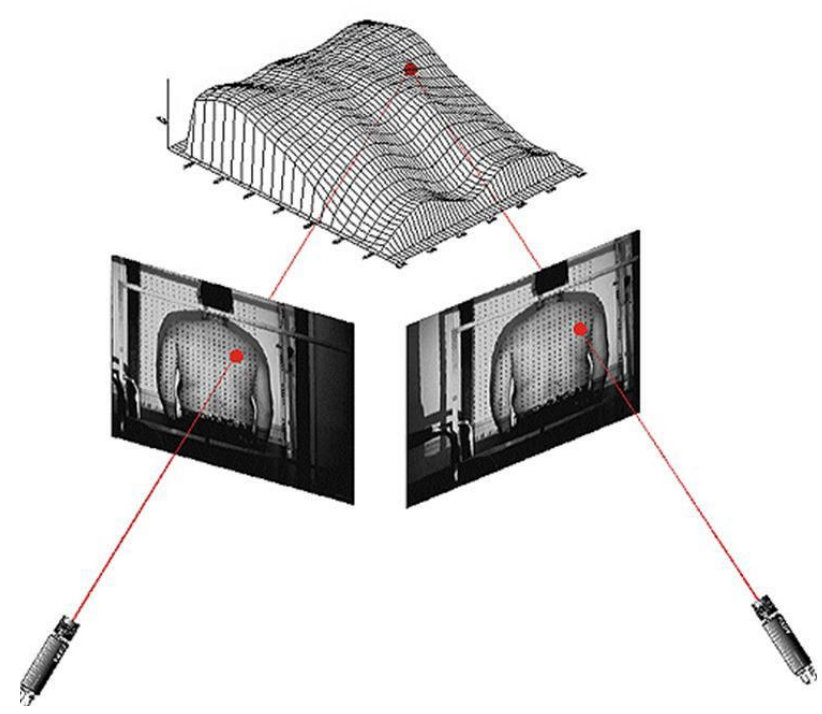

Figure 12. Image registration from the different sources; Source: (Patias et al., 2006)

\section{DISCUSSION AND FUTURE WORK}

In this work, authors reviewed the papers of other researchers in the field of scoliosis and were led to some very useful conclusions, most important of which is that there is plenty of room for further research in the field. More particularly, beginning with Cobb angle calculation, being the main metric for the severity of scoliosis, recent studies have tried to automatically compute it, but this computation included manual selection of the ROI and the final result greatly depended on the quality of the initial X-ray. On the other hand, it is proven that Cobb angle has its deficiencies, since it is a $2 \mathrm{D}$ metric for a $3 \mathrm{D}$ deformation. Researchers tried to evolve the scoliosis assessment by creating the 3D representation of the spine with various methodologies that have certain drawbacks: from using generalised information along with personalised data to utilising auxiliary items that limit the usability of the method. In addition, it was shown that excess use of radiation is harmful for the patient, especially the children who are the ones mostly diagnosed with scoliosis, forcing the community to turn their research in radiation-free scoliosis assessment methodologies.

A logical next step after carefully reviewing the current state of the art, is an integrated tool that is able to automatically perform a complete and accurate scoliosis assessment and monitoring. Authors plan to design a platform that takes as input an X-ray and a 3D picture of the patient's back, merging them into a 3D model of the spine, on which various Cobb angles are going to be calculated automatically with much more precision than the manual measuring. 3D Cobb angles can also be automatically computed, allowing more accurate monitoring of the 3D deformity of the spine. Moreover, a minimization of X-rays could be achieved through a one-to-one transformation algorithm from the X-ray to the 3D image of the back that could allow X-ray prediction through the low-cost and no-radiation 3D images of the back.

\section{ACKNOWLEDGEMENTS}

This paper is part of an ongoing post-doctoral research that is funded by the IKY-SIEMENS scholarship in Greece, for the period of September 2014.

\section{REFERENCES}

Benameur, S., Mignotte, M., Labelle, H., and De Guise, J. A., 2005. A Hierarchical Statistical Modeling Approach for the Unsupervised 3D Biplanar Reconstruction of the Scoliotic Spine IEEE Transactions On Biomedical Engineering, Vol. 52, No. 12.

Benjelloun, M., and Mahmoudi, S., 2009. A Hierarchical Statistical Modeling Spine Localization in X-ray Images Using Interest Point Detection, J Digit Imaging. June; 22(3): 309-318.

Canny, J., A., 1986, Computational Approach To Edge Detection, IEEE Trans. Pattern Analysis and Machine Intelligence, 8(6):679-698

Delorme, S., Petit, Y., De Guise, J. A., Labelle, H., Aubin, C.E., and Dansereau, J., 2003. Assessment of the 3-D Reconstruction and High-Resolution Geometrical Modelling of the Human Skeletal Trunk From 2-D Radiographic Images. IEEE Transactions On Biomedical Engineering, Vol. 50, No. 8.

Duong, L., Cheriet, F., and Labelle, H., 2010. Automatic Detection of Scoliotic Curves in Posteroanterior Radiographs, IEEE Transactions On Biomedical Engineering, Vol. 57, No. 5.

Frerich, J.M., Hertzler, K., Knott, P., and Mardjetko, S., 2012, Comparison of Radiographic and Surface Topography Measurements in Adolescents with Idiopathic Scoliosis, Open Orthop J. 2012; 6: 261-265. Published online 2012 July 27. doi: $10.2174 / 1874325001206010261$

Kadoury, S., Cheriet, F., Laporte, C., Labelle, H., 2007, A versatile $3 \mathrm{D}$ reconstruction system of the spine and pelvis for clinical assessment of spinal deformities, Med Bio Eng Comput 45:591-602

Kadoury, S., Cheriet, F., Labelle, H., 2009, Personalized X-Ray 3-D Reconstruction of the Scoliotic Spine From Hybrid Statistical and Image-Based Models, IEEE Transactions On Medical Imaging, Vol. 28, No. 9

Kadoury, S., Cheriet F., Labelle H., 2010, Self-Calibration of Biplanar Radiographic Images Through Geometric Spine Shape Descriptors, IEEE Transactions On Biomedical Engineering, Vol. 57, No. 7

Knott, P., Pappo, E., Cameron, M., deMauroy, JC., Rivard, C., Kotwicki, T., Zaina, F., Wynne, J., Stikeleather, L., BettanySaltikov, J., Grivas, Th., Durmala, J., Maruyama, T., Negrini, S., O’Brien, J.P., and Rigo, M., 2012, SOSORT 2012 consensus paper: reducing $\mathrm{X}$-ray exposure in pediatric patients with scoliosis, http://www.scoliosisjournal.com/content/9/1/4, Scoliosis 2014, 9:4

Kundu, R., Chakrabarti, A., Lenka, P. K., 2012, Cobb Angle Measurement of Scoliosis with Reduced Variability, MedImage2012, Computer Vision and Pattern Recognition arXiv:1211.5355 
Lawrence, A. and Rinsky, 2010, Quantifying scoliosis: we are still not there, The Spine Journal 10 (2010) 813-814

Markelj, P., Tomaževič, D., Likar, B. and Pernuš, F., 2012. A review of $3 \mathrm{D} / 2 \mathrm{D}$ registration methods for image-guided interventions. Medical Image Analysis Vol. 16 Issue 3, 642661

Patias, P., Stylianidis, E., Pateraki, M., Chrysanthou, Y., Contozis, C., Zavitsanakis, Th., 2006, 3D digital photogrammetric reconstructions for scoliosis screening, ISPRS Archives - Volume XXXVI Part 5, Proceedings of the ISPRS Commission V Symposium, 'Image Engineering and Vision Metrology'

Sardjono, T. A., Wilkinson, M. H. F., Veldhuizen, A. G., van Ooijen, P. M. A. Purnama, K. E., Verkerke, G. J., 2013, Automatic Cobb Angle Determination From Radiographic Images, Spine: 15 September 2013 - Volume 38 - Issue 20 - p E1256-E1262 doi: 10.1097/BRS.0b013e3182a0c7c3,

Diagnostics

Zhang, J., Lou, E., Le L.H., Hill, D. L., Raso, J. V., and Wang, Y., 2009, Automatic Cobb Measurement of Scoliosis Based on Fuzzy Hough Transform with Vertebral Shape Prior, Journal of Digital Imaging, Vol 22, No 5: pp 463 Y 472

\section{APPENDIX}

AIS: Adolescent Idiopathic Scoliosis AP: Anterior Posterior

DLT: Direct Linear Transformation

FFD: Free Form Deformation

FHT: Fuzzy Hough Transform

LAT: Lateral

PA_0: Posterior Anterior in zero degrees

ROI: Region of Interest

SVM: Support Vector Machines 